\title{
Risk factors for increased left ventricular hypertrophy in patients with chronic kidney disease: findings from the CKD-JAC study
}

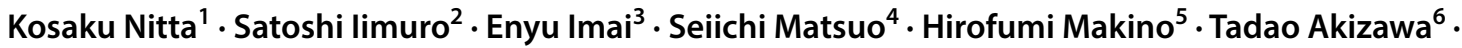 \\ Tsuyoshi Watanabe $^{7} \cdot$ Yasuo Ohashi $^{8} \cdot$ Akira Hishida9 $^{9}$
}

Received: 15 January 2018 / Accepted: 19 June 2018 / Published online: 27 June 2018

(c) The Author(s) 2018

\begin{abstract}
Background Although left ventricular hypertrophy (LVH) has been established as a predictor of cardiovascular events in chronic kidney disease (CKD), the relationship between the prevalence of LVH and CKD stage during the pre-dialysis period has not been fully examined.

Methods We measured left ventricular mass index (LVMI) in a cross-sectional cohort of participants in the Chronic Kidney Disease Japan Cohort (CKD-JAC) study to identify factors that are associated with increased LVMI in patients with stage 3-5 CKD.

Results We analyzed the baseline characteristics in 1088 participants (male 63.8\%, female $36.2 \%$ ). Diabetes mellitus was the underlying disease in $41.7 \%$ of the patients, and mean age was $61.8 \pm 11.1$ years. $\mathrm{LVH}$ was detected in $23.4 \%$ of the patients at baseline. By multivariate logistic analysis, independent risk factors for LVH were past history of cardiovascular disease [odds ratio (OR) 2.364; 95\% confidence interval ([CI) 1.463-3.822; $P=0.0004$ ], body mass index (OR $1.108 ; 95 \%$ CI 1.046-1.173; $P=0.0005$ ), systolic blood pressure (OR 1.173; 95\% CI 1.005-1.369; $P=0.0433$ ), urinary albumin (OR $1.425 ; 95 \%$ CI 1.028-1.974; $P=0.0333$ ), and serum total cholesterol level (OR 0.994; 95\% CI 0.989-0.999; $P=0.0174$ ).

Conclusion The cross-sectional baseline data from the CKD-JAC study shed light on the association between LVH and risk factors in patients with decreased renal function. Further longitudinal analyses of the CKD-JAC cohort are needed to evaluate the prognostic value of LVH in CKD patients.
\end{abstract}

Keywords Chronic kidney disease $\cdot$ Left ventricular hypertrophy $\cdot$ Hypertension $\cdot$ Body mass index $\cdot$ Albuminuria $\cdot$ Mineral metabolism $\cdot$ Antihypertensive agent

Kosaku Nitta

knitta@twmu.ac.jp

1 Department of Medicine, Kidney Center, Tokyo Women's Medical University, Tokyo 162-8666, Japan

2 Teikyo Academic Research Center, Teikyo University, Tokyo, Japan

3 Nakayamadera Imai Clinic, Hyogo, Japan

4 Department of Nephrology, Nagoya University, Aichi, Japan

5 Okayama University, Okayama, Japan

6 Division of Nephrology, Department of Medicine, Showa University School of Medicine, Tokyo, Japan

7 Japan Organization of Occupational Health and Safety Fukushima Rosai Hospital, Fukushima, Japan

8 Department of Integrated Science and Engineering for Sustainable Society, Chuo University, Tokyo, Japan

9 Yaizu City Hospital, Shizuoka, Japan

\section{Introduction}

Chronic kidney disease (CKD) is the leading risk factor for cardiovascular disease (CVD), a great threat to health and an economic burden [1]. In Japan, the prevalence of end-stage kidney disease (ESKD) requiring renal replacement therapy has been increasing over the last three decades. There were 38,327 new cases in 2014 , bringing the total number of cases in Japan to 320,448 [2]. Since the number of patients requiring dialysis has continued to increase [3], there appears to be an enormous number of latent cases of CKD in the Japanese population. In a recent study, Imai et al. reported the prevalence of CKD by calculating the estimated glomerular filtration rate (eGFR) using an equation that estimates GFR based on data from the Japanese annual health check programing 2005 [4]. They predicted that $13 \%$ of the Japanese adult population (approximately 13.3 million people) 
would have CKD in 2005. Mortality, predominantly due to cardiovascular events, is high in patients with CKD and left ventricular hypertrophy $(\mathrm{LVH})$ is a strong risk factor [5].

Renal dysfunction and albuminuria in CKD patients have been established as a risk factor for cardiovascular (CV) events independent of conventional CV risk factors [6-9]. Population-based studies in Western and Asian countries have shown that the risk of CVD increases as renal function declines. Because of this finding, the National Kidney Foundation formed a task force to heighten awareness of CVD in CKD, and defined CKD using parameters such as decreased eGFR $<60 \mathrm{ml} / \mathrm{min} / 1.73 \mathrm{~m}^{2}$. A cohort of CKD patients closely observed by the physicians is required to accurately analyze renal and CV events. However, few studies have been conducted on the prevalence of LVH in a predialysis population [10-13].

The aim of the present study was to clarify whether there is a close correlation between the prevalence of $\mathrm{LVH}$ and the stage of CKD classified according to eGFR and to identify factors related to $\mathrm{LVH}$ among the participants in the Chronic Kidney Disease Japan Cohort (CKD-JAC) [14].

\section{Subjects and methods}

\section{Inclusion and exclusion criteria}

Baseline characteristics of CKD-JAC are described elsewhere [15]. The following inclusion criteria were used at screening: (1) Japanese or Asian patients living in Japan; (2) age 20-75 years; and (3) a broad spectrum of CKD with eGFR of $10-59 \mathrm{ml} / \mathrm{min} / 1.73 \mathrm{~m}^{2}$. eGFR was calculated using a modified three-variable equation for eGFR in Japanese patients [16]: eGFR $=194 \times \mathrm{age}^{-0.287} \times \mathrm{sCr}^{-1.094}(\times 0.739$, if female), where $\mathrm{sCr}=$ serum creatinine.

All patients were classified on the basis of CKD stage as described in our previous paper [14]. The following patients were excluded from participation: (1) patients with polycystic kidney disease, human immunodeficiency virus (HIV) infection, liver cirrhosis, active cancer, and patients who had received cancer treatment within the past 2 years; (2) transplant recipients and patients who had previously been on long-term dialysis; (3) patients who refused to provide informed consent.

Information on past medical history, including hypertension, acute myocardial infarction, angina pectoris, congestive heart failure, peripheral arterial disease, cerebrovascular disease, and prescription of antihypertensive agents, including angiotensin-converting enzyme (ACE) inhibitors, angiotensin receptor blockers (ARBs), calcium channel blockers (CCBs), diuretics, and b-blockers, statins, and antiplatelet agents, was collected from the medical records at each institution.

\section{Blood pressure and echocardiographic measurements}

Blood pressure (BP) was measured in outpatient clinics with an automated sphygmomanometer after a 5-min rest. $\mathrm{BP}$ in the right arm was measured three times at intervals of $1 \mathrm{~min}$, and the mean values were used for analyses. A mercury sphygmomanometer was used to measure the BP of patients who had frequent premature contractions, atrial fibrillation, or atrial flutter. Pulse pressure was calculated by subtracting diastolic BP from systolic BP. A two-dimensional guided M-mode echocardiographic study was performed in 1178 patients at each institution. Of them, the cases whose left ventricular end-diastolic diameter was not measured nor body surface area could not be calculated were excluded. Finally, this study included 1088 cases. Measurements included the diastolic thickness of the interventricular septum (IVST) and left ventricular posterior wall (PWT), and the internal diameter of the left ventricle at the end of diastole (LVDd) and the end of systole (LVDs). The modified Penn cube formula was used to calculate LV mass [17]: $1.04 \times[(0.1 \times$ IVST $)+(0.1 \times$ PWT $)] \times 3-[(0.1 \times$ LVDd $) \times 3$ ]$\times 0.8+0.6$, and LV mass was adjusted for body surface area (LVMI). LVH was defined as LVMI $>125 \mathrm{~g} / \mathrm{m}^{2}$ in men and $>110 \mathrm{~g} / \mathrm{m}^{2}$ in women [18]. Relative wall thickness (RWT) was calculated at end diastole as 2PWTd/LVDd and considered to be increased if $>0.45$. The LV geometry was categorized as follows: normal (no LVH and normal RW), eccentric hypertrophy (LVH and normal RWT), and concentric hypertrophy (LVH and increased RWT).

\section{Definitions of hypertension, diabetes, and dyslipidemia}

Hypertension was defined as systolic $\mathrm{BP} \geq 140 \mathrm{mmHg}$ and/or diastolic $\mathrm{BP} \geq 90 \mathrm{mmHg}$ or taking an antihypertensive agent. Diabetes mellitus (DM) was defined as $\mathrm{HbA} 1 \mathrm{C} \geq 6.5 \%$ or taking an antidiabetic agent. Diabetic patients were identified as those with diabetic nephropathy as the primary cause of CKD. Dyslipidemia was defined as serum triglyceride level $>150 \mathrm{mg} / \mathrm{dl}$, or serum high-density lipoprotein (HDL) cholesterol level $<40 \mathrm{mg} / \mathrm{dl}$ in men and $<50 \mathrm{mg} / \mathrm{dl}$ in women.

\section{Collection of biological samples and measurements}

Whole blood, serum, and urine samples were collected for measurement of serum $\mathrm{Cr}, \mathrm{HbAlc}$, intact parathyroid hormone (iPTH), and urinary albumin and $\mathrm{Cr}$ levels at a central laboratory. Urinary albumin excretion was expressed as the albumin to $\mathrm{Cr}$ ratio (ACR). HbA1c was measured by the 
JDS method, and the value was converted to the A1C value measured by the NGSP method by adding $0.4 \%$ as determined by the Japanese Diabetes Society. Each clinical center measured serum $\mathrm{Cr}$ at each visit. A 24-h urine specimen was collected from each patient once a year to measure the amount of proteinuria.

\section{Statistical analysis}

All variables are reported as mean $\pm \mathrm{SD}$ and frequency. Descriptive statistics of baseline characteristics were calculated by CKD stage, sex, and the presence or absence of LVH. CKD stages were defined according to the patient's eGFR. Chi-squared test and Student's $t$ test or one-way analysis of variance (ANOVA) were used to detect betweengroup differences. ACR values had a skewed distribution and were log-transformed to achieve a normal distribution. Logistic linear regression was used to investigate the relation of LVMI to eGFR, BMI, and log ACR. Univariate logistic regression analyses were performed in an attempt to identify factors related to LVH. Multivariate logistic regression analyses were used to identify independent variables related to $L V H$. We considered some variables that had a $P$ value $<0.10$ in univariate logistic regression analyses as independent variables for multivariate logistic regression analyses. The model included the variables as follows: sex, smoking status, complications of DM, dyslipidemia and hypertension, past history of congestive heart failure (CHF), angina pectoris, myocardial infarction (MI), stroke and peripheral artery disease (PAD), systolic and diastolic BPs, pulse pressure, BMI, eGFR, uric acid, ACR, A1C, iPTH, HDL cholesterol, triglyceride, calcium, phosphorus, and prescription of antihypertensive agents. The two-sided $95 \%$ confidence interval (CI) and odds ratio (OR) were calculated by estimation. A two-sided probability level of 5\% was considered significant. All statistical analyses were performed using the SAS software program for Windows ver. 9.4 (SAS Inc. Japan, Tokyo, Japan).

\section{Results}

\section{Baseline demographics and clinical characteristics of participants according to eGFR level}

We studied 2966 participants. Eleven participants (1 liver cirrhosis, 8 cancers, and 2 others) were excluded from 2977 of baseline participants [13]. The baseline characteristics of the 2966 participants in the CKD-JAC study have been described previously [14]. Of them, the subjects in this study, i.e., those $(N=1088)$ who were examined by echocardiography (UCG), consisted of 694 Japanese men (63.8\%) and 394 Japanese women (36.2\%), 454 (41.7\%) and 807
(74.2\%) of whom had DM and dyslipidemia, respectively. Most of the subjects had hypertension (88.7\%) and were being treated with an antihypertensive agent $(92.5 \%)$, most of them with ACE inhibitors (25.8\%)/ARBs (75.9\%), as shown in Table 1.

CKD was stage $3 \mathrm{a}$ in 128 patients $(11.8 \%)$, stage $3 \mathrm{~b}$ in 349 patients (32.1\%), stage 4 in 427 patients (39.2\%), and stage 5 in 184 patients $(16.9 \%)$ (Table 1$)$. The prevalence of CVD comorbidity tended to be inversely proportional to eGFR, but the correlation did not reach statistical significance. The groups with stage 4-5 CKD were older and had higher systolic BP and pulse pressure, a higher prevalence of hyperuricemia and anemia, and higher grades of proteinuria and albuminuria than the groups with stage $3 a$ and $3 b$ CKD, and serum levels of phosphorus and iPTH in stage 4 and 5 CKD patients were significantly higher than those in stage $3 a$ and $3 b$ CKD patients. The frequency of antihypertensive agents, including CCBs and diuretics, was gradually increased in accordance with the progression of CKD stage.

\section{Analysis by sex}

Since the proportion of male subjects was $63.8 \%$ in the study population, sex may have affected the results of the present study. As shown in Table 2, female subjects were younger and had a lower prevalence of hypertension, DM, and past history of MI and stroke than male subjects. In addition, female subjects had lower BMI, lower serum levels of $\mathrm{Cr}$ and uric acid, and lower hemoglobin concentration than male subjects. However, there was no significant sex difference in eGFR. Female subjects had higher serum levels of lipids, including total cholesterol, non-HDL cholesterol, low-density lipoprotein (LDL) cholesterol, and HDL cholesterol, and lower serum triglyceride level. Lower percentages of female subjects were prescribed antihypertensive agents, including $\mathrm{ARB}, \mathrm{CCB}$ s and $\beta$-blockers, statins and antiplatelet agents. As shown in Table 5, menopause was not significantly associated with LVMI by univariate logistic regression analyses.

\section{Comparison of the study population with and without LVH according to CKD stage and sex}

LVMI in each of the four groups of CKD patients according to eGFR is shown in Fig. 1 and tended to increase with the stage of CKD $(P=0.0005$ in men, $P=0.0016$ in women). The prevalence of eccentric and concentric LVH was higher among patients with more advanced CKD stages (G3a: 7.8 vs. $7.8 \%$, G3b: 8.9 vs. $9.2 \%$, G4: 10.8 vs. $14.3 \%$, G5: $14.7 \%$ vs. $20.7 \%)$. The prevalence of LVH was 255 of $1088(23.4 \%)$ of the study population (Table 3$)$. Men had a higher prevalence of LVH than women (25.1 vs. 20.6\%). 
Table 1 Baseline characteristics of the study population by eGFR

\begin{tabular}{|c|c|c|c|c|c|c|}
\hline \multirow[t]{2}{*}{ Variable } & \multirow[t]{2}{*}{ All patients } & \multicolumn{4}{|c|}{ eGFR (ml/min/1.73 m2) } & \multirow[t]{2}{*}{$P$ Value } \\
\hline & & Stage $3 a \geq 45$ & Stage $3 b 30$ to $<45$ & Stage 415 to $<30$ & Stage $5<15$ & \\
\hline$N$ & 1088 & 128 & 349 & 427 & 184 & \\
\hline Age (years) & $61.8 \pm 11.1$ & $56.4 \pm 12.9$ & $61.6 \pm 11.2$ & $62.7 \pm 10.6$ & $63.7 \pm 9.4$ & $<0.0001$ \\
\hline \multicolumn{7}{|l|}{$\operatorname{Sex}[n(\%)]$} \\
\hline Male & $694(63.8)$ & $82(64.1)$ & $228(65.3)$ & $273(63.9)$ & $111(60.3)$ & 0.724 \\
\hline \multicolumn{7}{|l|}{ Medical history $[n(\%)]$} \\
\hline Hypertension & $965(88.7)$ & $105(82.0)$ & $298(85.4)$ & $397(93.0)$ & $165(89.7)$ & 0.0006 \\
\hline Diabetes & $454(41.7)$ & $54(42.2)$ & $142(40.7)$ & $178(41.7)$ & $80(43.5)$ & 0.9405 \\
\hline Dyslipidemia & $807(74.2)$ & $97(75.8)$ & $255(73.1)$ & $319(74.7)$ & $136(73.9)$ & 0.9262 \\
\hline \multicolumn{7}{|l|}{ Cardiovascular disease } \\
\hline MI & $71(6.5)$ & $7(5.5)$ & $20(5.7)$ & $29(6.8)$ & $15(8.2)$ & 0.6954 \\
\hline Angina & $113(10.4)$ & $9(7.0)$ & $35(10.0)$ & $45(10.5)$ & $24(13.0)$ & 0.3912 \\
\hline Congestive heart failure & $60(5.5)$ & $4(3.1)$ & $18(5.2)$ & $25(5.9)$ & $13(7.1)$ & 0.4877 \\
\hline PAD & $40(3.7)$ & $3(2.3)$ & $9(2.6)$ & $18(4.2)$ & $10(5.4)$ & 0.2855 \\
\hline Stroke & $134(12.3)$ & $17(13.3)$ & $43(12.3)$ & $49(11.5)$ & $25(13.6)$ & 0.8814 \\
\hline $\mathrm{BMI}\left(\mathrm{kg} / \mathrm{m}^{2}\right)$ & $23.6 \pm 3.8$ & $24.1 \pm 3.3$ & $23.7 \pm 3.9$ & $23.5 \pm 3.8$ & $23.4 \pm 3.6$ & 0.3595 \\
\hline \multicolumn{7}{|l|}{ Blood pressure $(\mathrm{mmHg})$} \\
\hline Systolic & $132.7 \pm 18.2$ & $130.4 \pm 17.2$ & $129.7 \pm 17.7$ & $133.7 \pm 18.2$ & $137.8 \pm 18.3$ & $<0.0001$ \\
\hline Diastolic & $76.0 \pm 12.0$ & $76.0 \pm 10.8$ & $75.1 \pm 11.8$ & $76.3 \pm 12.1$ & $77.0 \pm 12.7$ & 0.2876 \\
\hline Pulse pressure (mmHg) & $56.7 \pm 14.0$ & $54.4 \pm 14.1$ & $54.7 \pm 13.5$ & $57.4 \pm 14.0$ & $60.7 \pm 13.8$ & $<0.0001$ \\
\hline Creatinine (mg/dl) & $2.17 \pm 1.07$ & $1.10 \pm 0.18$ & $1.43 \pm 0.24$ & $2.31 \pm 0.53$ & $4.02 \pm 0.85$ & $<0.0001$ \\
\hline $\mathrm{eGFR}\left(\mathrm{mL} / \mathrm{min} / 1.73 \mathrm{~m}^{2}\right)$ & $28.7 \pm 12.7$ & $50.8 \pm 5.3$ & $37.3 \pm 4.2$ & $22.4 \pm 4.3$ & $11.9 \pm 1.9$ & $<0.001$ \\
\hline Uric acid (mg/dl) & $7.24 \pm 1.52$ & $6.51 \pm 1.41$ & $7.013 \pm 1.35$ & $7.47 \pm 1.56$ & $7.65 \pm 1.58$ & $<0.001$ \\
\hline Urinary protein (g/day) & $1.6 \pm 2.2$ & $0.8 \pm 1.8$ & $1.3 \pm 2.1$ & $1.7 \pm 2.2$ & $2.4 \pm 2.2$ & $<0.0001$ \\
\hline Urinary albumin $(\mathrm{mg} / \mathrm{g} \mathrm{Cr})$ & $1064.4 \pm 1475.8$ & $538.9 \pm 967.6$ & $881.2 \pm 1615.2$ & $1164.5 \pm 1375.2$ & $1544.8 \pm 1556.8$ & $<0.0001$ \\
\hline Total chol (mg/dl) & $195.0 \pm 43.8$ & $199.3 \pm 36.5$ & $198.4 \pm 48.0$ & $195.0 \pm 41.2$ & $185.9 \pm 44.7$ & 0.0152 \\
\hline Non-HDL chol (mg/dl) & $141.1 \pm 42.2$ & $141.5 \pm 36.7$ & $143.0 \pm 45.8$ & $142.0 \pm 39.5$ & $135.4 \pm 44.6$ & 0.3141 \\
\hline LDL chol (mg/dl) & $111.4 \pm 34.3$ & $116.2 \pm 28.5$ & $113.1 \pm 38.3$ & $110.8 \pm 32.2$ & $106.3 \pm 34.2$ & 0.0814 \\
\hline HDL chol (mg/dl) & $54.2 \pm 18.3$ & $58.1 \pm 19.0$ & $56.1 \pm 19.1$ & $53.0 \pm 18.0$ & $50.8 \pm 16.2$ & 0.0014 \\
\hline Triglyceride (mg/dl) & $170.7 \pm 117.2$ & $165.9 \pm 141.9$ & $166.0 \pm 111.3$ & $177.0 \pm 123.0$ & $168.6 \pm 94.2$ & 0.5993 \\
\hline Calcium (mg/dl) & $9.0 \pm 0.56$ & $9.3 \pm 0.4$ & $9.1 \pm 0.5$ & $9.0 \pm 0.5$ & $8.7 \pm 0.6$ & $<0.0001$ \\
\hline Phosphorus (mg/dl) & $3.5 \pm 0.7$ & $3.3 \pm 0.6$ & $3.3 \pm 0.6$ & $3.6 \pm 0.6$ & $4.1 \pm 0.8$ & $<0.0001$ \\
\hline iPTH (pg/ml) & $104.9 \pm 82.8$ & $54.8 \pm 24.0$ & $66.6 \pm 35.2$ & $106.6 \pm 58.8$ & $207.2 \pm 121.0$ & $<0.0001$ \\
\hline $\mathrm{CRP}(\mathrm{mg} / \mathrm{dl})$ & $0.3 \pm 1.0$ & $0.1 \pm 0.2$ & $0.2 \pm 0.5$ & $0.3 \pm 0.8$ & $0.4 \pm 1.9$ & 0.1071 \\
\hline $\mathrm{A} 1 \mathrm{C}(\%)$ & $6.0 \pm 1.0$ & $6.0 \pm 1.0$ & $6.1 \pm 1.1$ & $5.9 \pm 0.9$ & $5.9 \pm 0.8$ & 0.0227 \\
\hline Hemoglobin (g/dl) & $12.2 \pm 1.9$ & $13.3 \pm 1.8$ & $13.0 \pm 1.80$ & $11.7 \pm 1.5$ & $10.8 \pm 1.4$ & $<0.0001$ \\
\hline \multicolumn{7}{|l|}{ Medication $[n(\%)]$} \\
\hline Antihypertensive agent & $1006(92.5)$ & 107 (83.6) & $320(91.7)$ & 404 (94.6) & $175(95.1)$ & 0.0002 \\
\hline ARB & $826(75.9)$ & $93(72.7)$ & 257 (73.6) & 337 (78.9) & $139(75.5)$ & 0.2771 \\
\hline ACEI & $281(25.8)$ & $24(18.8)$ & $100(28.7)$ & $126(29.5)$ & $31(16.8)$ & 0.0014 \\
\hline CCB & $627(57.6)$ & $57(44.5)$ & $174(49.9)$ & $271(63.5)$ & $125(67.9)$ & $<0.0001$ \\
\hline$\beta$-Blocker & $290(26.7)$ & $26(20.3)$ & $71(20.3)$ & $131(30.7)$ & $62(33.7)$ & 0.0005 \\
\hline Statin & $468(43.0)$ & $64(50.0)$ & $151(43.3)$ & 177 (41.5) & $76(41.3)$ & 0.3615 \\
\hline Diuretic & $371(34.1)$ & $24(18.8)$ & $108(30.9)$ & $161(37.7)$ & $78(42.4)$ & $<0.0001$ \\
\hline Antiplatelet & $280(25.7)$ & $23(18.0)$ & 94 (26.9) & $107(25.1)$ & $56(30.4)$ & 0.0885 \\
\hline
\end{tabular}

$M I$ myocardial infarction, $P A D$ peripheral artery disease, $B M I$ body mass index, $C h o l$ cholesterol, $L D L$ low-density lipoprotein, $H D L$ highdensity lipoprotein, $i P T H$ intact parathyroid hormone, $C R P$ C-reactive protein, $A R B$ angiotensin receptor blocker, $A C E I$ angiotensin-converting enzyme inhibitor, $C C B$ calcium channel blocker 
Table 2 Baseline characteristics of the study population by sex

\begin{tabular}{|c|c|c|c|c|}
\hline \multirow[t]{2}{*}{ Variable } & \multirow[t]{2}{*}{ All patients } & \multicolumn{2}{|l|}{ Sex } & \multirow[t]{2}{*}{$P$ Value } \\
\hline & & Female & Male & \\
\hline$N$ & 1088 & $394(36.2)$ & $694(63.8)$ & $<0.0001$ \\
\hline Age (years) & $61.8 \pm 11.1$ & $60.9 \pm 11.7$ & $62.3 \pm 10.7$ & 0.0402 \\
\hline \multicolumn{5}{|l|}{ Medical history $[n(\%)]$} \\
\hline Hypertension & $965(88.7)$ & $335(85.0)$ & $630(90.8)$ & 0.004 \\
\hline Diabetes & $454(41.7)$ & $147(37.3)$ & $307(44.2)$ & 0.026 \\
\hline Dyslipidemia & $807(74.2)$ & $297(75.4)$ & $510(73.5)$ & 0.4928 \\
\hline \multicolumn{5}{|l|}{ Cardiovascular disease } \\
\hline MI & $71(6.5)$ & $8(2.0)$ & $63(9.1)$ & $<0.0001$ \\
\hline Angina & $113(10.4)$ & $25(6.3)$ & $88(12.7)$ & 0.001 \\
\hline Congestive heart failure & $60(5.5)$ & $17(4.3)$ & $43(6.2)$ & 0.1914 \\
\hline PAD & $40(3.7)$ & $9(2.3)$ & $31(4.5)$ & 0.066 \\
\hline Stroke & $134(12.3)$ & $31(7.9)$ & $103(14.8)$ & 0.0008 \\
\hline BMI $\left(\mathrm{kg} / \mathrm{m}^{2}\right)$ & $23.6 \pm 3.8$ & $23.1 \pm 4.1$ & $23.9 \pm 3.5$ & 0.0014 \\
\hline \multicolumn{5}{|l|}{ Blood pressure $(\mathrm{mmHg})$} \\
\hline Systolic & $132.7 \pm 18.2$ & $131.5 \pm 18.9$ & $133.4 \pm 17.7$ & 0.0834 \\
\hline Diastolic & $76.0 \pm 12.0$ & $74.8 \pm 12.0$ & $76.7 \pm 11.9$ & 0.0119 \\
\hline Pulse pressure (mmHg) & $56.7 \pm 14.0$ & $56.6 \pm 14.4$ & $56.8 \pm 13.7$ & 0.880 \\
\hline Creatinine (mg/dl) & $2.17 \pm 1.07$ & $1.85 \pm 0.88$ & $2.36 \pm 1.13$ & $<0.0001$ \\
\hline eGFR (ml/min/1.73 m²) & $28.7 \pm 12.7$ & $28.4 \pm 13.0$ & $28.9 \pm 12.5$ & 0.5329 \\
\hline Uric acid (mg/dl) & $7.2 \pm 1.5$ & $6.9 \pm 1.5$ & $7.4 \pm 1.5$ & $<0.001$ \\
\hline Urinary protein (g/day) & $1.6 \pm 2.171$ & $1.3 \pm 2.0$ & $1.7 \pm 2.3$ & 0.0874 \\
\hline Urinary albumin (mg/gCr) & $1064.1 \pm 1475.8$ & $1008.9 \pm 1552.0$ & $1095.8 \pm 1430.4$ & 0.3583 \\
\hline Total chol (mg/dl) & $195.0 \pm 43.8$ & $208.4 \pm 45.7$ & $187.3 \pm 40.7$ & $<0.0001$ \\
\hline Non-HDL chol (mg/dl) & $141.1 \pm 42.2$ & $148.3 \pm 44.8$ & $137.1 \pm 40.2$ & 0.0001 \\
\hline LDL chol (mg/dl) & $111.4 \pm 34.3$ & $118.7 \pm 35.3$ & $107.3 \pm 33.1$ & $<0.0001$ \\
\hline HDL chol (mg/dl) & $54.2 \pm 18.3$ & $61.2 \pm 19.2$ & $50.3 \pm 16.6$ & $<0.0001$ \\
\hline Triglyceride (mg/dl) & $170.7 \pm 117.2$ & $160.7 \pm 107.3$ & $176.4 \pm 122.2$ & 0.0414 \\
\hline Calcium (mg/dl) & $9.03 \pm 0.55$ & $9.15 \pm 0.54$ & $8.96 \pm 0.55$ & $<0.0001$ \\
\hline Phosphorus (mg/dl) & $3.5 \pm 0.7$ & $3.80 \pm 0.6$ & $3.4 \pm 0.7$ & $<0.0001$ \\
\hline iPTH $(\mathrm{pg} / \mathrm{ml})$ & $104.9 \pm 82.8$ & $109.5 \pm 85.7$ & $102.3 \pm 81.0$ & 0.1717 \\
\hline $\mathrm{CRP}$ (mg/dl) & $0.3 \pm 1.0$ & $0.2 \pm 0.4$ & $0.3 \pm 1.2$ & 0.1370 \\
\hline $\mathrm{A} 1 \mathrm{C}(\%)$ & $6.0 \pm 1.0$ & $6.0 \pm 1.0$ & $6.0 \pm 0.9$ & 0.9776 \\
\hline Hemoglobin (g/dl) & $12.1 \pm 1.9$ & $11.5 \pm 1.6$ & $12.51 \pm 1.9$ & $<0.0001$ \\
\hline \multicolumn{5}{|l|}{ Medication $[n(\%)]$} \\
\hline Antihypertensive agent & $1006(92.5)$ & $351(89.1)$ & $655(94.4)$ & 0.0015 \\
\hline ARB & $826(75.9)$ & $284(72.1)$ & $542(78.1)$ & 0.0257 \\
\hline ACEI & $281(25.8)$ & $101(25.6)$ & $180(25.9)$ & 0.9129 \\
\hline CCB & $627(57.6)$ & $206(52.3)$ & $421(60.7)$ & 0.0072 \\
\hline$\beta$-Blocker & $290(26.7)$ & $90(22.8)$ & $200(28.8)$ & 0.0321 \\
\hline Statin & $468(43.0)$ & $201(51.0)$ & $267(38.5)$ & $<0.0001$ \\
\hline Diuretic & $371(34.1)$ & $131(33.2)$ & $240(34.6)$ & 0.6557 \\
\hline Antiplatelet & $280(25.7)$ & $66(16.8)$ & $214(30.8)$ & $<0.0001$ \\
\hline
\end{tabular}

The demographic and biochemical parameters of the study population are compared in Table 4. Female subjects with $\mathrm{LVH}$ had a higher prevalence of DM, past history of MI, and $\mathrm{CHF}$ and stroke, higher systolic BP, higher pulse pressure, lower eGFR, and higher ACR than female subjects without LVH. In addition, female subjects with LVH had lower serum HDL cholesterol level, lower calcium level, and higher serum levels of phosphorus and iPTH than female subjects without LVH. Moreover, female subjects with LVH had higher serum CRP value and lower hemoglobin concentration, when compared with female subjects without LVH. Finally, higher proportions of female subjects with 


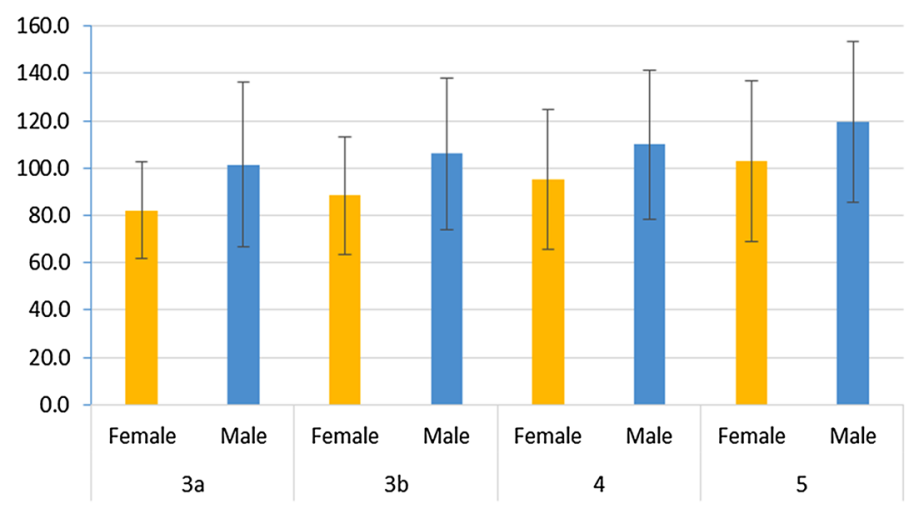

\begin{tabular}{ccccc}
\hline \multirow{2}{*}{ CKD stage } & Sex & Number & Mean & \multicolumn{1}{l}{ SD } \\
& & & & \\
\hline \multirow{2}{*}{$3 a$} & Female & 46 & 82.2 & 20.3 \\
& Male & 82 & 101.6 & 34.7 \\
\hline \multirow{2}{*}{$3 b$} & Female & 121 & 88.4 & 24.9 \\
\cline { 2 - 5 } & Male & 228 & 106.2 & 32.1 \\
\hline \multirow{2}{*}{4} & Female & 154 & 95.2 & 29.4 \\
\cline { 2 - 5 } & Male & 273 & 110.0 & 31.6 \\
\hline \multirow{2}{*}{5} & Female & 73 & 102.9 & 33.8 \\
\cline { 2 - 5 } & Male & 111 & 119.3 & 34.1 \\
\hline
\end{tabular}

Fig. 1 Relationship between estimated glomerular filtration rate (eGFR) and left ventricular mass index (LVMI) of patients with stage 3-5 CKD. a Female, b male

LVH were being treated with CCBs, $\beta$-blockers, diuretics, and antiplatelet drugs.

On the other hand, higher proportions of male subjects with LVH had hypertension and DM, and higher proportions had past history of MI, angina and CHF, when compared with male subjects without LVH. The group of male subjects with LVH had higher BMI, higher systolic BP, higher pulse pressure, lower eGFR, and higher ACR than female subjects without LVH. Among the lipid parameters, male subjects with LVH had significantly lower serum levels of HDL cholesterol level, when compared with male subjects without LVH. Parameters of mineral metabolism and hemoglobin concentration showed the same trends in female subjects as in male subjects with LVH. Moreover, higher proportions of male with LVH were being treated with antihypertensive agents similar to those in female subjects with LVH. LVMI was negatively associated with eGFR (Fig. 2), whereas LVMI was positively associated with BMI (Fig. 3).

\section{Factors related to $\mathrm{LVH}$}

Table 5 shows that the factors associated with LVH were age, DM, and hypertension. Past history of CVD except peripheral artery disease was significantly related to LVH. Significant clinical factors associated with LVH were systolic BP, pulse pressure, eGFR, BMI, ACR, serum levels of calcium, phosphorus, and iPTH, total cholesterol, HDL cholesterol, hemoglobin concentration, and prescription of erythropoiesis-stimulating agents and antiplatelet drugs.

As shown in Table 6, the variables independently associated with LVH were past history of CVD, systolic blood pressure, BMI, urinary albumin, and serum total cholesterol level by multivariate logistic regression analysis.

As shown in Table 7, the variable independently associated with LVH in diabetic patients was only HDL cholesterol by multivariate logistic regression analysis. BMI has also some kind of relationship with LVH, but not significant. As shown in Table 8 , the variables independently associated with LVH in non-diabetic CKD patients were past history of CVD, BMI, and total cholesterol by multivariate logistic regression analysis. Systolic BP and hemoglobin have also some kind of relationship with LVH, but not significant. Patients with eccentric and concentric LVH had significant risk factors with a history of previous CVD and higher BMI (Table 9).

\section{Discussion}

In the present cross-sectional study, we enrolled 2966 representative Japanese outpatients, most of whom had stage 3-5 CKD. These 2966 outpatients were being treated by nephrologists and were receiving a good standard of care. UCG was performed in 1088 of them. The UCG carried out was not intended to evaluate selected patients with cardiac complications, but was performed consecutively for evaluation of cardiac function in representative participants in the CKD-JAC study, if they provided informed consent. The prevalence (23.4\%) of LVH in the present study was much lower than $(30 \%<)$ that reported in previous studies in the pre-dialysis CKD population [19-21]. The participants in the CKD-JAC study may be better treated by nephrologists. Alternatively, cardiologists could treat more severe cases. The majority of the study subjects had hypertension and proteinuria or albuminuria on enrollment, but systolic and diastolic BP were prehypertensive (132/76 mmHg).

More than $90 \%$ of the subjects were being treated with antihypertensive agents $(n=1006,92.5 \%)$, including ACE inhibitors $(n=281,25.8 \%)$ and/or ARBs $(n=826,75.9 \%)$. The prevalence rates of pre-existing CVD, i.e., MI (6.5\%), angina (10.4\%), CHF (5.5\%), peripheral artery disease $(3.7 \%)$, and stroke $(12.3 \%)$, were higher than in the general Japanese population [22]. DM was present in $41.7 \%$ of the study subjects, and more than one-third of enrolled 
Table 3 Baseline characteristics of the study population by LVH

\begin{tabular}{|c|c|c|c|c|}
\hline \multirow[t]{2}{*}{ Variable } & \multirow[t]{2}{*}{ All patients } & \multicolumn{2}{|l|}{ LVH } & \multirow[t]{2}{*}{$P$ Value } \\
\hline & & LVH (-) & LVH (+) & \\
\hline$N$ & 1088 & 833 & 255 & \\
\hline Age (years) & $61.8 \pm 11.1$ & $61.2 \pm 11.3$ & $63.8 \pm 9.9$ & 0.0007 \\
\hline Sex (male, \%) & $694(63.8)$ & $520(62.4)$ & $174(68.2)$ & 0.0912 \\
\hline \multicolumn{5}{|l|}{ Medical history $[n(\%)]$} \\
\hline Hypertension & 965 (88.7) & $727(87.3)$ & $238(93.3)$ & 0.0075 \\
\hline Diabetes & $454(41.7)$ & $322(38.7)$ & $132(51.8)$ & 0.0002 \\
\hline Dyslipidemia & 807 (74.2) & $615(73.8)$ & $192(75.3)$ & 0.6401 \\
\hline \multicolumn{5}{|l|}{ Cardiovascular disease } \\
\hline MI & $71(6.5)$ & $39(4.7)$ & $32(12.5)$ & $<0.0001$ \\
\hline Angina & $113(10.4)$ & $71(8.5)$ & $42(16.5)$ & 0.0003 \\
\hline Congestive heart failure & $60(5.5)$ & $32(3.8)$ & $28(11.0)$ & $<0.0001$ \\
\hline PAD & $40(3.7)$ & $28(3.4)$ & $12(4.7)$ & 0.3181 \\
\hline Stroke & $134(12.3)$ & $90(10.8)$ & $44(17.3)$ & 0.0061 \\
\hline BMI $\left(\mathrm{kg} / \mathrm{m}^{2}\right)$ & $23.6 \pm 3.8$ & $23.4 \pm 3.8$ & $24.4 \pm 3.6$ & 0.0002 \\
\hline \multicolumn{5}{|l|}{ Blood pressure (mmHg) } \\
\hline Systolic & $132.7 \pm 18.2$ & $131.4 \pm 17.4$ & $137.0 \pm 19.9$ & $<0.0001$ \\
\hline Diastolic & $76.0 \pm 12.0$ & $76.1 \pm 11.6$ & $75.7 \pm 13.2$ & 0.6413 \\
\hline Pulse pressure $(\mathrm{mmHg})$ & $56.7 \pm 14.0$ & $55.3 \pm 13.1$ & $61.3 \pm 15.8$ & $<0.0001$ \\
\hline Creatinine (mg/dl) & $2.2 \pm 1.1$ & $2.1 \pm 1.0$ & $2.5 \pm 1.2$ & $<0.0001$ \\
\hline eGFR (ml/min/1.73 m²) & $28.7 \pm 12.7$ & $29.8 \pm 12.6$ & $25.2 \pm 12.4$ & $<0.0001$ \\
\hline Uric acid (mg/dl) & $7.2 \pm 1.5$ & $7.2 \pm 1.5$ & $7.4 \pm 1.5$ & 0.0517 \\
\hline Urinary protein (mg/day) & $1.6 \pm 2.2$ & $1.4 \pm 2.1$ & $2.1 \pm 2.5$ & 0.0108 \\
\hline Urinary albumin (mg/gCr) & $1064.1 \pm 1475.8$ & $934.5 \pm 1378.1$ & $1490.0 \pm 1693.5$ & $<0.0001$ \\
\hline Total chol (mg/dl) & $195.0 \pm 43.8$ & $196.8 \pm 43.1$ & $189.1 \pm 45.6$ & 0.0195 \\
\hline Non-HDL chol (mg/dl) & $141.1 \pm 42.2$ & $141.7 \pm 42.1$ & $139.2 \pm 42.7$ & 0.4682 \\
\hline LDL chol (mg/dl) & $111.4 \pm 34.3$ & $111.3 \pm 34.1$ & $110.6 \pm 35.1$ & 0.8984 \\
\hline HDL chol (mg/dl) & $54.2 \pm 18.3$ & $55.5 \pm 18.9$ & $49.7 \pm 15.6$ & $<0.0001$ \\
\hline Triglyceride (mg/dl) & $170.7 \pm 117.2$ & $170.6 \pm 121.1$ & $171.2 \pm 103.5$ & 0.9444 \\
\hline Calcium (mg/dl) & $9.0 \pm 0.6$ & $9.1 \pm 0.5$ & $8.9 \pm 0.6$ & $<0.0001$ \\
\hline Phosphorus (mg/dl) & $3.5 \pm 0.7$ & $3.5 \pm 0.7$ & $3.7 \pm 0.7$ & 0.0003 \\
\hline ¡PTH (pg/ml) & $104.9 \pm 82.8$ & $98.7 \pm 79.2$ & $125.5 \pm 90.9$ & $<0.0001$ \\
\hline CRP (mg/dl) & $0.3 \pm 1.0$ & $0.2 \pm 0.5$ & $0.4 \pm 1.8$ & 0.0052 \\
\hline $\mathrm{A} 1 \mathrm{C}(\%)$ & $6.0 \pm 1.0$ & $6.0 \pm 0.9$ & $6.1 \pm 1.0$ & 0.2286 \\
\hline Hemoglobin (g/dl) & $12.2 \pm 1.9$ & $12.3 \pm 1.8$ & $11.7 \pm 2.1$ & $<0.0001$ \\
\hline \multicolumn{5}{|l|}{ Medication $[n(\%)]$} \\
\hline Antihypertensive agent & $1006(92.5)$ & $766(92.0)$ & $240(94.1)$ & 0.2527 \\
\hline $\mathrm{ARB}$ & $826(75.9)$ & $635(76.2)$ & $191(74.9)$ & 0.6642 \\
\hline ACEI & $281(25.8)$ & $209(25.1)$ & $72(28.2)$ & 0.3153 \\
\hline $\mathrm{CCB}$ & $627(57.6)$ & $452(54.3)$ & $175(68.6)$ & $<0.0001$ \\
\hline$\beta$-Blocker & $290(26.7)$ & $181(21.7)$ & $109(42.7)$ & $<0.0001$ \\
\hline Statin & $468(43.0)$ & $359(43.1)$ & 109 (42.7) & 0.9208 \\
\hline Diuretic & $371(34.1)$ & $256(30.7)$ & $115(45.1)$ & $<0.0001$ \\
\hline Antiplatelet & $280(25.7)$ & $171(20.5)$ & 109 (42.7) & $<0.0001$ \\
\hline
\end{tabular}

subjects had CKD secondary to glomerulonephritis. Subgroup analysis of LVMI and related factors of non-diabetic patients showed that history of previous CVD, BMI, and total cholesterol was significant and systolic BP and hemoglobin were almost significant (Table 8). However, those in diabetic patients showed that only HDL cholesterol was significant. Diabetes is considered to be a strong predictor for $\mathrm{LVH}$, especially concentric $\mathrm{LVH}$, in CKD patients as recently described [23, 24]. 
Table 4 Baseline characteristics of the study population by sex and LVH

\begin{tabular}{|c|c|c|c|c|c|c|}
\hline \multirow[t]{2}{*}{ Variable } & \multicolumn{2}{|l|}{ Female } & \multirow[t]{2}{*}{$P$ value } & \multicolumn{2}{|l|}{ Male } & \multirow[t]{2}{*}{$P$ value } \\
\hline & $\mathrm{LVH}(-)(-)$ & LVH (+) & & $\mathrm{LVH}(-)$ & LVH $(+)$ & \\
\hline$N$ & 313 & 81 & & 520 & 174 & \\
\hline Age (years) & $60.0 \pm 11.9$ & $64.2 \pm 10.1$ & 0.004 & $61.8 \pm 10.9$ & $63.7 \pm 9.8$ & 0.0506 \\
\hline \multicolumn{7}{|l|}{ Medical history $[n(\%)]$} \\
\hline Hypertension & $267(85.3)$ & $68(84.0)$ & 0.761 & $460(88.5)$ & $170(97.7)$ & 0.0003 \\
\hline Diabetes & $107(34.2)$ & $40(49.4)$ & 0.0117 & $215(41.3)$ & $92(52.9)$ & 0.008 \\
\hline Dyslipidemia & $234(74.8)$ & $63(77.8)$ & 0.5742 & $381(73.3)$ & $129(74.1)$ & 0.8222 \\
\hline \multicolumn{7}{|l|}{ Cardiovascular disease } \\
\hline MI & $4(1.3)$ & $4(4.9)$ & 0.0374 & $35(6.7)$ & $28(16.1)$ & 0.0002 \\
\hline Angina & $17(5.4)$ & $8(9.9)$ & 0.1435 & $54(10.4)$ & $34(19.5)$ & 0.0017 \\
\hline Congestive heart failure & $10(3.2)$ & $7(8.6)$ & 0.0315 & $22(4.2)$ & $21(12.1)$ & 0.0002 \\
\hline ASO & $6(1.9)$ & $3(3.7)$ & 0.3374 & $22(4.2)$ & $9(5.2)$ & 0.6027 \\
\hline Stroke & $16(5.1)$ & $15(18.5)$ & $<0.0001$ & $74(14.2)$ & $29(16.7)$ & 0.434 \\
\hline BMI (kg/m2) & $23.0 \pm 4.1$ & $23.6 \pm 4.0$ & 0.2269 & $23.6 \pm 3.5$ & $24.7 \pm 3.4$ & 0.0002 \\
\hline \multicolumn{7}{|l|}{ Blood pressure $(\mathrm{mmHg})$} \\
\hline Systolic & $130.3 \pm 18.3$ & $136.0 \pm 20.6$ & 0.0155 & $132.1 \pm 16.9$ & $137.5 \pm 19.7$ & 0.0005 \\
\hline Diastolic & $74.8 \pm 11.6$ & $74.9 \pm 13.9$ & 0.9353 & $76.9 \pm 11.5$ & $76.1 \pm 12.9$ & 0.4245 \\
\hline Pulse pressure (mmHg) & $55.5 \pm 14.0$ & $60.9 \pm 15.2$ & 0.0028 & $55.2 \pm 12.5$ & $61.4 \pm 16.1$ & $<0.001$ \\
\hline Creatinine (mg/dl) & $1.8 \pm 0.8$ & $2.2 \pm 1.0$ & $<0.0001$ & $2.3 \pm 1.1$ & $2.6 \pm 1.2$ & 0.0007 \\
\hline eGFR (ml/min/1.73 m2) & $29.8 \pm 13.0$ & $22.9 \pm 11.5$ & $<0.0001$ & $29.8 \pm 12.3$ & $26.2 \pm 12.7$ & 0.001 \\
\hline Uric acid (mg/dl) & $6.9 \pm 156$ & $7.0 \pm 1.4$ & 0.376 & $7.4 \pm 1.5$ & $7.6 \pm 1.5$ & 0.1369 \\
\hline Urinary protein (mg/day) & $1.2 \pm 2.0$ & $1.7 \pm 1.7$ & 0.2266 & $1.5 \pm 2.1$ & $2.2 \pm 2.7$ & 0.0338 \\
\hline Urinary albumin (mg/gCr) & $865.8 \pm 1466.4$ & $1559.3 \pm 1748.6$ & 0.0004 & $976.1 \pm 1321.4$ & $1457.1 \pm 1671.0$ & 0.0002 \\
\hline Total chol (mg/dl) & $210.2 \pm 43.9$ & $201.5 \pm 51.9$ & 0.1405 & $188.7 \pm 40.5$ & $183.0 \pm 41.0$ & 0.1325 \\
\hline Non-HDL chol (mg/dl) & $148.7 \pm 44.7$ & $146.9 \pm 45.6$ & 0.7778 & $137.5 \pm 40.0$ & $135.7 \pm 40.9$ & 0.6332 \\
\hline LDL chol (mg/dl) & $118.5 \pm 34.8$ & $119.5 \pm 37.6$ & 0.8396 & $107.0 \pm 33.0$ & $108.0 \pm 33.4$ & 0.7398 \\
\hline HDL chol (mg/dl) & $62.5 \pm 19.4$ & $55.6 \pm 17.7$ & 0.0082 & $51.3 \pm 17.3$ & $47.1 \pm 13.8$ & 0.0062 \\
\hline Triglyceride (mg/dl) & $160.0 \pm 113.4$ & $163.1 \pm 79.5$ & 0.8263 & $176.9 \pm 125.2$ & $174.9 \pm 112.9$ & 0.86 \\
\hline Calcium (mg/dl) & $9.2 \pm 0.5$ & $9.0 \pm 0.6$ & 0.0012 & $9.0 \pm 0.5$ & $8.8 \pm 0.6$ & 0.0001 \\
\hline Phosphorus (mg/dl) & $3.8 \pm 0.6$ & $4.0 \pm 0.7$ & 0.0005 & $3.3 \pm 0.7$ & $3.5 \pm 0.7$ & 0.0038 \\
\hline iPTH (pg/ml) & $102.7 \pm 78.0$ & $135.5 \pm 106.9$ & 0.0022 & $96.2 \pm 79.9$ & $120.6 \pm 82.0$ & 0.0007 \\
\hline $\mathrm{CRP}(\mathrm{mg} / \mathrm{dl})$ & $0.2 \pm 0.4$ & $0.3 \pm 0.5$ & 0.0122 & $0.3 \pm 0.6$ & $0.5 \pm 2.2$ & 0.0353 \\
\hline $\mathrm{A} 1 \mathrm{C}(\%)$ & $6.0 \pm 1.0$ & $6.1 \pm 0.9$ & 0.2007 & $6.0 \pm 0.9$ & $6.0 \pm 1.0$ & 0.5995 \\
\hline Hemoglobin (g/dl) & $11.7 \pm 1.5$ & $10.9 \pm 1.7$ & $<0.0001$ & $12.7 \pm 1.8$ & $12.1 \pm 2.1$ & 0.0006 \\
\hline \multicolumn{7}{|l|}{ Medication $[n(\%)]$} \\
\hline Antihypertensive agent & $277(88.5)$ & $74(91.4)$ & 0.4619 & $489(94.0)$ & $166(95.4)$ & 0.4989 \\
\hline ARB & $230(73.5)$ & $54(66.7)$ & 0.2229 & 405 (77.9) & 137 (78.7) & 0.8142 \\
\hline ACEI & $76(24.3)$ & $25(30.9)$ & 0.2265 & $133(25.6)$ & $47(27.0)$ & 0.7086 \\
\hline $\mathrm{CCB}$ & $154(49.2)$ & $52(64.2)$ & 0.016 & $298(57.3)$ & $123(70.7)$ & 0.0018 \\
\hline$\beta$-Blocker & $60(19.2)$ & $30(37.0)$ & 0.0006 & $121(23.3)$ & $79(45.4)$ & $<0.0001$ \\
\hline Statin & $159(50.8)$ & $42(51.9)$ & 0.8658 & $200(38.5)$ & $67(38.5)$ & 0.9917 \\
\hline Diuretic & $92(29.4)$ & $39(48.1)$ & 0.0014 & $164(31.5)$ & $76(43.7)$ & 0.0036 \\
\hline Antiplatelet & $38(12.1)$ & $28(34.6)$ & $<0.0001$ & $133(25.6)$ & $81(46.6)$ & $<0.00$ \\
\hline
\end{tabular}

The results of the present study provided information on the prevalence of LVH and factors associated with LVH in stage 3-5 CKD patients in the CKD-JAC study. In the CKDJAC study, LVH was observed in a small population (23.4\%) of the 1088 study subjects, whereas LVMI tended to increase with the progression of CKD. In addition, the prevalence of eccentric and concentric LVH was higher among patients with more advanced CKD stages. CKD patients have a high prevalence of $\mathrm{LVH}$, ranging from 34 to $74 \%$ in different studies, and its prevalence increases as renal function 
a LVMI vs eGFR(ml/min/1.73m2) male

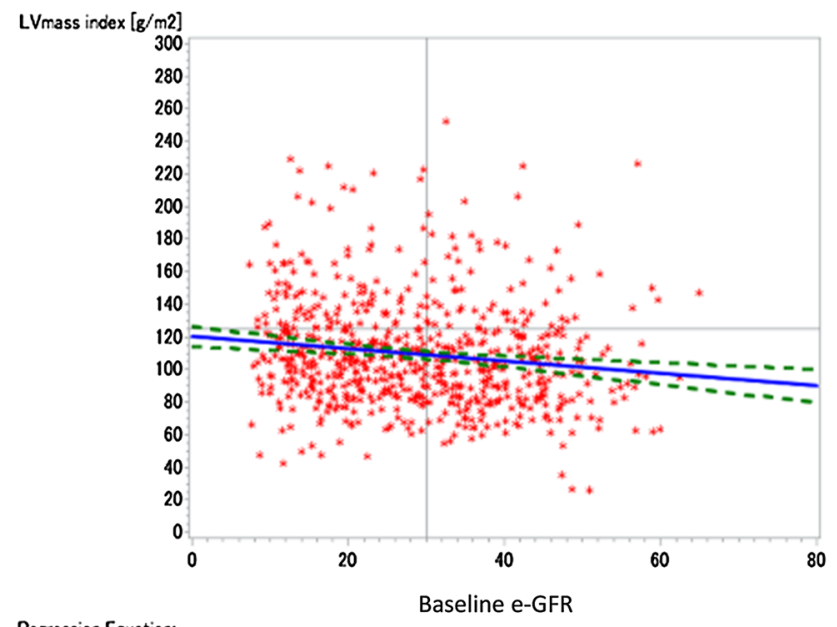

Regression Equation:
LVhII $=120.1406-0.377945 * b$ EGFR b LVMI vs eGFR(ml/min/1.73m2) female

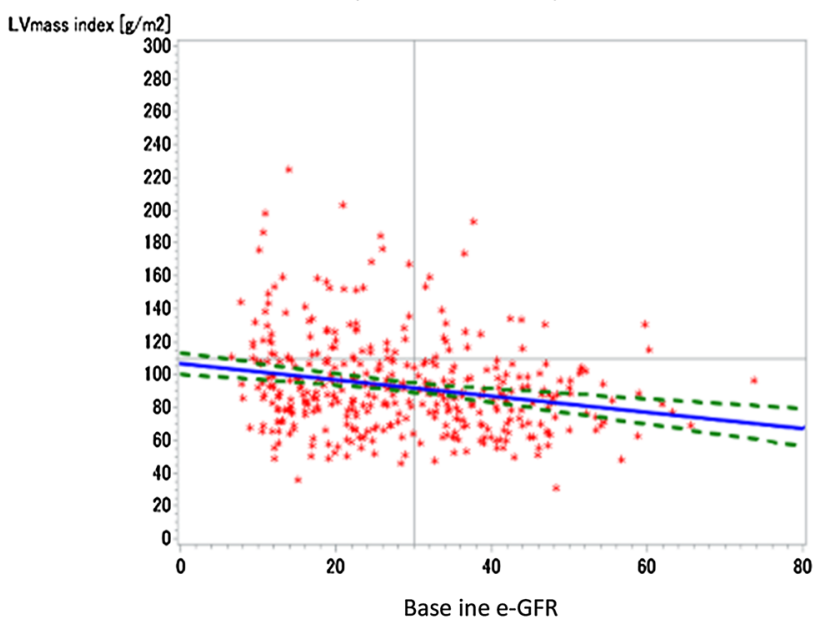

Regression Equation:
LVMII $=106.8445-0.487406 * b$ EEGR

Fig. 2 Relationship between body mass index (BMI) and left ventricular mass index (LVMI) of patients with stage 3-5 CKD. a Female; b male
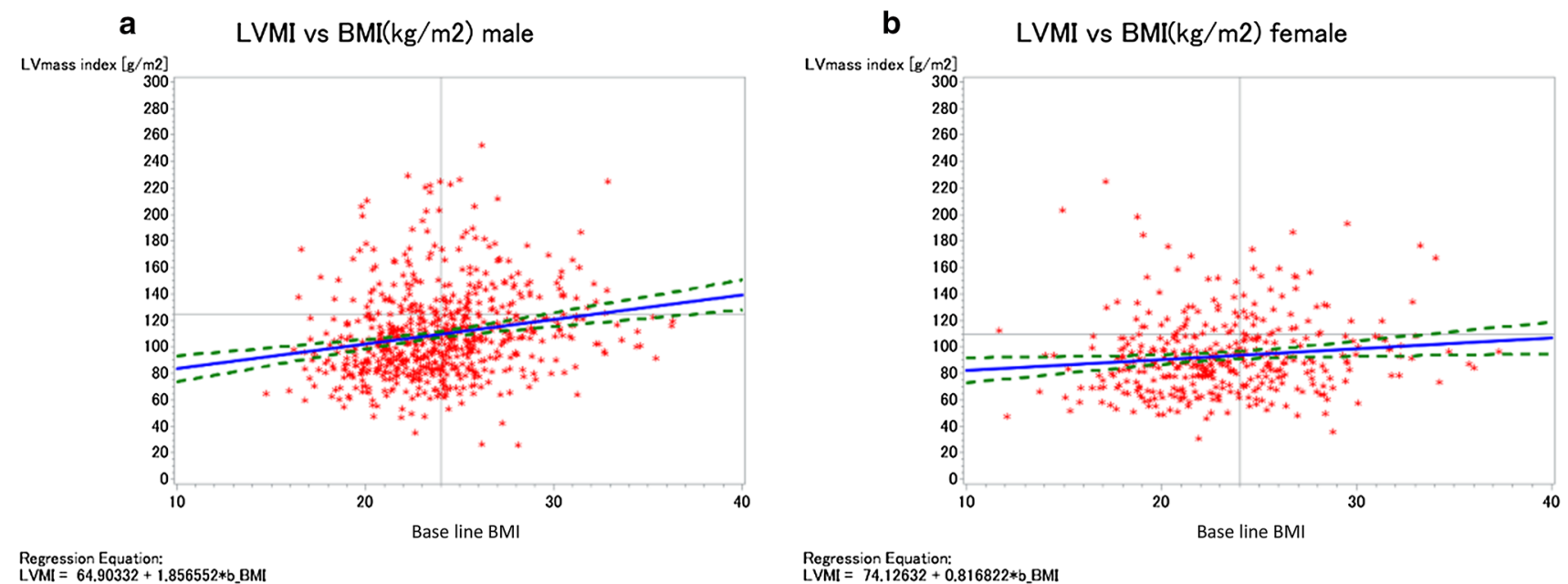

Fig. 3 Comparison of left ventricular mass index (LVMI) in the different subgroups of CKD patients according to their degree of renal dysfunction

declines [11, 13, 25, 26]. However, the relatively wide heterogeneity of the prevalence of LVH in different studies can be attributed to several differences in the characteristics of the populations studied, including differences in ethnicity, age, proportion of subjects with different stages of CKD, prevalence of hypertension, method chosen to evaluate GFR, cutoff GFR used to enroll patients, and definition of LVH.

Elevated systolic BP has a continuous, graded, and independent association with risk of coronary heart disease, stroke, and ESKD [27]. LVH might be a beneficial compensatory process in CKD patients, allowing the left ventricle to produce additional force to increase cardiac work and maintain constant wall tension [28]. Even though mean systolic
BP was well controlled $(132.7 \pm 18.2 \mathrm{mmHg})$, systolic BP was higher in patients with LVH than in patients without LVH in the present study. According to multivariate logistic regression analysis, systolic BP was an independent variable associated with LVH. Recently, it was reported that systolic arterial hypertension and elevated pulse pressure are closely associated with LVH in pre-dialysis patients, suggesting that fluid overload and increased arterial stiffness play important roles in LVH before starting dialysis therapy [13]. From the pathophysiologic standpoint, an increase in afterload induces concentric LVH, whereas volume overload leads to concentric LVH. In patients with CKD, the simultaneous coexistence of all factors (hypertension, arterial stiffness, volume 
Table 5 Factors associated with LVMI (univariate logistic regression analysis)

\begin{tabular}{|c|c|c|c|}
\hline Variables & OR & $95 \% \mathrm{CI}$ & $P$ value \\
\hline Sex (female) & 1.293 & $0.959-1.743$ & 0.0917 \\
\hline Age (years) & 1.024 & $1.010-1.039$ & 0.0008 \\
\hline Smoking & 1.06 & $0.707-1.589$ & 0.7771 \\
\hline Menopause & 1.327 & $0.906-1.944$ & 0.1467 \\
\hline \multicolumn{4}{|l|}{ Complications } \\
\hline Diabetes & 1.703 & $1.284-2.259$ & 0.0002 \\
\hline Dyslipidemia & 1.08 & $0.781-1.493$ & 0.6402 \\
\hline Hypertension & 2.225 & $1.046-4.732$ & 0.0377 \\
\hline \multicolumn{4}{|l|}{ Medical history } \\
\hline Cardiovascular disease & 2.477 & $1.848-3.320$ & $<0.0001$ \\
\hline MI & 2.921 & $1.789-4.771$ & $<0.0001$ \\
\hline Angina & 2.116 & $1.404-3.191$ & 0.0003 \\
\hline Congestive heart failure & 3.086 & $1.820-5.234$ & $<0.0001$ \\
\hline ASO & 1.42 & $0.711-2.835$ & 0.3201 \\
\hline Stroke & 1.722 & $1.164-2.547$ & 0.0066 \\
\hline \multicolumn{4}{|l|}{ Blood pressure $(\mathrm{mmHg})$} \\
\hline Systolic $(10 \mathrm{mmHg})$ & 1.182 & $1.094-1.277$ & $<0.0001$ \\
\hline Diastolic $(10 \mathrm{mmHg})$ & 0.972 & $0.864-1.094$ & 0.6409 \\
\hline Pulse pressure $(\mathrm{mmHg})$ & 1.03 & $1.020-1.040$ & $<0.0001$ \\
\hline $\mathrm{BMI}\left(\mathrm{kg} / \mathrm{m}^{2}\right)$ & 1.703 & $1.034-1.113$ & 0.0002 \\
\hline eGFR $\left(\mathrm{ml} / \mathrm{min} / 1.73 \mathrm{~m}^{2}\right)$ & 0.97 & $0.958-0.981$ & $<0.001$ \\
\hline Uric acid (mg/dl) & 1.096 & $0.999-1.202$ & 0.0521 \\
\hline Urinary albumin $(\mathrm{mg} / \mathrm{gCr})$ & 1.658 & $1.342-2.049$ & $<0.0001$ \\
\hline $\mathrm{A} 1 \mathrm{C}(\%)$ & 1.093 & $0.945-1.265$ & 0.2291 \\
\hline Hemoglobin (g/dl) & 0.835 & $0.771-0.905$ & $<0.0001$ \\
\hline iPTH (pg/ml) & 1.003 & $1.002-1.005$ & $<0.0001$ \\
\hline Total chol (mg/dl) & 0.996 & $0.992-0.999$ & 0.0199 \\
\hline Non-HDL chol (mg/dl) & 0.999 & $0.995-1.002$ & 0.4678 \\
\hline LDL chol (mg/dl) & 1 & $0.996-1.005$ & 0.898 \\
\hline HDL chol (mg/dl) & 0.98 & $0.971-0.990$ & $<0.0001$ \\
\hline Triglyceride (mg/dl) & 1 & $0.999-1.001$ & 0.9441 \\
\hline Calcium (mg/dl) & 0.505 & $0.387-0.658$ & $<0.001$ \\
\hline Phosphorus (mg/dl) & 1.456 & $1.182-1.792$ & 0.0004 \\
\hline \multicolumn{4}{|l|}{ Medication } \\
\hline Antihypertensive agent & 1.399 & $0.785-2.495$ & 0.2549 \\
\hline Statin & 0.986 & $0.742-1.309$ & 0.9209 \\
\hline ESA & 1.497 & $1.014-2.209$ & 0.0423 \\
\hline Phosphate binder & 0.266 & $0.062-1.131$ & 0.0729 \\
\hline Vitamin D & 0.968 & $0.568-1.648$ & 0.9035 \\
\hline Antiplatelet & 2.89 & $2.142-3.900$ & $<0.0001$ \\
\hline
\end{tabular}

$O R$ odds ratio, $C I$ confidence interval, $E S A$ erythropoiesis-stimulating agent

expansion, and anemia) may preclude the development of specific alterations in LV geometry because of an overlap of different hemodynamic stimuli. Fluid volume management and maintenance of a near euvolemic state are crucial for the amelioration of LVH [29].
Table 6 Factors associated with LVMI (multivariate logistic regression analysis)

\begin{tabular}{llll}
\hline Variables & OR & $95 \%$ CI & $P$ Value \\
\hline Sex (female) & 0.966 & $0.589-1.585$ & 0.8926 \\
Age (years) & 1.017 & $0.994-1.041$ & 0.1515 \\
Smoking & 1.021 & $0.593-1.76$ & 0.9400 \\
Hypertension & 0.695 & $0.25-1.932$ & 0.4857 \\
Cardiovascular disease & 2.364 & $1.463-3.822$ & 0.0004 \\
Stroke & 1.063 & $0.581-1.945$ & 0.8421 \\
Systolic $(10 \mathrm{mmHg})$ & 1.173 & $1.005-1.369$ & 0.0433 \\
Diastolic $(10 \mathrm{mmHg})$ & 0.895 & $0.708-1.132$ & 0.3544 \\
BMI $\left(\mathrm{kg} / \mathrm{m}^{2}\right)$ & 1.108 & $1.046-1.173$ & 0.0005 \\
eGFR (ml/min/1.73 m²) & 0.992 & $0.971-1.015$ & 0.5025 \\
Uric acid $(\mathrm{mg} / \mathrm{dL})$ & 1.024 & $0.898-1.168$ & 0.7240 \\
Urinaryalbumin(log mg/gCr) & 1.425 & $1.028-1.974$ & 0.0333 \\
Total chol $(\mathrm{mg} / \mathrm{dL})$ & 0.994 & $0.989-0.999$ & 0.0174 \\
HDL chol $(\mathrm{mg} / \mathrm{dL})$ & 0.989 & $0.976-1.003$ & 0.1375 \\
Calcium $(\mathrm{mg} / \mathrm{dL})$ & 0.83 & $0.549-1.255$ & 0.3781 \\
Phosphorus (mg/dL) & 0.95 & $0.687-1.314$ & 0.7561 \\
A1C $(\%)$ & 0.852 & $0.681-1.065$ & 0.1602 \\
Hemoglobin(g/dL) & 0.955 & $0.827-1.103$ & 0.5319 \\
iPTH(pg/mL) & 1.001 & $0.998-1.003$ & 0.5423 \\
\hline
\end{tabular}

$O R$ odds ratio, $C I$ confidence interval

Table 7 Factors associated with LVMI by diabetic CKD patients (multivariate logistic regression analysis)

\begin{tabular}{llll}
\hline Variables & OR & $95 \%$ CI & $P$ Value \\
\hline Sex (female) & 1.234 & $0.56-2.722$ & 0.6022 \\
Age (years) & 1.014 & $0.979-1.051$ & 0.4423 \\
Smoking & 0.85 & $0.401-1.802$ & 0.6713 \\
Hypertension & 0.504 & $0.088-2.887$ & 0.4420 \\
Cardiovascular disease & 1.5 & $0.761-2.956$ & 0.2421 \\
Stroke & 1.036 & $0.459-2.339$ & 0.9325 \\
Systolic $(10 \mathrm{mmHg})$ & 1.132 & $0.909-1.41$ & 0.2688 \\
Diastolic $(10 \mathrm{mmHg})$ & 0.851 & $0.601-1.205$ & 0.3633 \\
BMI (kg/m $\left.{ }^{2}\right)$ & 1.082 & $0.991-1.182$ & 0.0789 \\
eGFR (ml/min/1.73 m $\left.{ }^{2}\right)$ & 0.986 & $0.956-1.018$ & 0.3977 \\
Uric acid (mg/ dL) & 0.946 & $0.771-1.161$ & 0.5967 \\
Urinary albumin (log mg/gCr) & 1.464 & $0.906-2.366$ & 0.1195 \\
Total chol(mg/ dL) & 0.996 & $0.989-1.004$ & 0.3196 \\
HDL chol (mg/ dL) & 0.971 & $0.947-0.996$ & 0.0252 \\
Calcium (mg/ dL) & 0.753 & $0.431-1.315$ & 0.3184 \\
Phosphorus (mg/ dL) & 1.192 & $0.746-1.903$ & 0.4633 \\
A1C (\%) & 0.84 & $0.633-1.115$ & 0.2284 \\
Hemoglobin(g/ dL) & 1.095 & $0.891-1.344$ & 0.3883 \\
iPTH (pg/mL) & 1.001 & $0.997-1.006$ & 0.4784 \\
\hline
\end{tabular}

$O R$ odds ratio, $C I$ confidence interval 
Table 8 Factors associated with LVMI by non-diabetic CKD patients (multivariate logistic regression analysis)

\begin{tabular}{llll}
\hline Variables & OR & $95 \%$ CI & $P$ Value \\
\hline Sex (female) & 0.661 & $0.332-1.313$ & 0.2367 \\
Age (years) & 1.029 & $0.995-1.064$ & 0.0948 \\
Smoking & 1.082 & $0.464-2.523$ & 0.8558 \\
Hypertension & 0.837 & $0.218-3.214$ & 0.7958 \\
Cardiovascular disease & 3.968 & $1.915-8.219$ & 0.0002 \\
Stroke & 1.05 & $0.41-2.691$ & 0.9184 \\
Systolic $(10 \mathrm{mmHg})$ & 1.254 & $0.98-1.604$ & 0.0719 \\
Diastolic $(10 \mathrm{mmHg})$ & 0.876 & $0.614-1.251$ & 0.4669 \\
BMI $\left(\mathrm{kg} / \mathrm{m}^{2}\right)$ & 1.146 & $1.053-1.246$ & 0.0015 \\
eGFR $\left(\mathrm{ml} / \mathrm{min} / 1.73 \mathrm{~m}^{2}\right)$ & 1.008 & $0.975-1.041$ & 0.6490 \\
Uric acid $(\mathrm{mg} / \mathrm{dL})$ & 1.086 & $0.906-1.302$ & 0.3716 \\
Urinary albumin $(\mathrm{log} \mathrm{mg} / \mathrm{gCr})$ & 1.372 & $0.843-2.233$ & 0.2028 \\
Total chol (mg/dL) & 0.991 & $0.983-0.999$ & 0.0244 \\
HDL chol (mg/dL) & 1.001 & $0.983-1.019$ & 0.9148 \\
Calcium $(\mathrm{mg} / \mathrm{d} \mathrm{dL})$ & 1.03 & $0.522-2.033$ & 0.9317 \\
Phosphorus $(\mathrm{mg} / \mathrm{dL})$ & 0.704 & $0.432-1.148$ & 0.1594 \\
A1C $(\%)$ & 0.632 & $0.293-1.363$ & 0.2421 \\
Hemoglobin $(\mathrm{g} / \mathrm{dL})$ & 0.812 & $0.656-1.004$ & 0.0547 \\
iPTH (pg/mL) & 1.001 & $0.998-1.005$ & 0.4324 \\
\hline
\end{tabular}

$O R$ odds ratio, $C I$ confidence interval

Table 9 Factors associated with eccentric and concentric LVH in CKD patients (multivariate logistic regression analysis)

\begin{tabular}{lllll}
\hline Variables & $\begin{array}{l}\text { Eccentric } \\
\text { LVH OR }\end{array}$ & $P$ value & $\begin{array}{l}\text { Concen- } \\
\text { tric LVH } \\
\text { OR }\end{array}$ & $P$ Value \\
& & & & \\
\hline Sex (male) & 1.011 & 0.976 & 0.950 & 0.873 \\
Age (years) & 1.030 & 0.111 & 1.014 & 0.332 \\
Smoking & 0.696 & 0.430 & 1.171 & 0.628 \\
Hypertension & 1.577 & 0.667 & 0.417 & 0.138 \\
Cardiovascular disease & 2.386 & 0.011 & 2.235 & 0.008 \\
Stroke & 0.844 & 0.702 & 1.238 & 0.562 \\
Systolic $(10 \mathrm{mmHg})$ & 1.199 & 0.114 & 1.141 & 0.176 \\
Diastolic $(10 \mathrm{mmHg})$ & 0.825 & 0.271 & 0.960 & 0.784 \\
BMI (kg/m $\left.{ }^{2}\right)$ & 1.111 & 0.011 & 1.098 & 0.008 \\
eGFR (ml/min/1.73 m²) & 1.010 & 0.542 & 0.982 & 0.193 \\
Uric acid (mg/dL) & 0.929 & 0.448 & 1.098 & 0.264 \\
Urinary albumin (log mg/ & 1.373 & 0.190 & 1.447 & 0.075 \\
gCr) & & & & \\
Total chol(mg/dL) & 0.955 & 0.235 & 0.928 & 0.020 \\
HDL chol (mg/dL) & 0.952 & 0.617 & 0.857 & 0.097 \\
Calcium (mg/dL) & 0.823 & 0.566 & 0.882 & 0.618 \\
Phosphorus (mg/dL) & 0.857 & 0.527 & 1.043 & 0.837 \\
A1C $(\%)$ & 0.822 & 0.257 & 0.851 & 0.252 \\
Hemoglobin(g/dL) & 0.802 & 0.051 & 1.060 & 0.517 \\
iPTH (pg/mL) & 1.002 & 0.178 & 1.000 & 0.958 \\
\hline
\end{tabular}

$O R$ odds ratio, $C I$ confidence interval
In accordance with the theory of non-hemodynamic LVH-promoting factors in our CKD patients, BMI was found to be a factor that was independently associated with LVH. Obesity is thought to be a risk factor independent of $\mathrm{LVH}$, and heart disorders in obesity include structural adaptation with LVH and functional abnormalities [30]. Kotsis et al. [31] reported that obesity and daytime pulse pressure are predictors of LVH in true normotensive individuals. In hypertensive obese patients, metabolic syndrome (MetS) maintains its role as a risk factor for LVH independently of age and systolic BP and is a useful predictor of target organ damage in clinical practice [32]. However, MetS is no longer an independent risk factor when BMI is taken into account, suggesting that the effects of MetS on LVH are mainly driven by the degree of abdominal adiposity.

Currently, information about sex differences in renal abnormalities and CVD in healthy individuals is limited and conflicting. In the Prevention of Renal and Vascular End-Stage Disease (PREVEND) study, the prevalence of microalbuminuria in men was almost double that observed in women, and for a higher value of age and BMI was greater in men than in women [33]. In addition, the presence of CKD has been found to be associated with an increased risk of cardiovascular events [34] and of cardiovascular death [35] in both women and men having different degrees of cardiovascular risk or already having CVD. A recent study has shown that logistic regression analysis demonstrated that the factors significantly associated with the prevalence of $\mathrm{LVH}$ were age and BMI in women and uric acid in men [36]. In the present study, sex difference was not significantly associated with LVH in diabetic- and non-diabetic CKD patients. In our cohort, men had a higher prevalence of classical CV risk factors including hypertension, past history of previous CVD, hyperuricemia, and lower HDL cholesterol, suggesting that classical $\mathrm{CV}$ risk factors may be associated with $\mathrm{LVH}$ in men with non-diabetic CKD.

The results of the present study have shown that albuminuria is an independent predictor of LVH in CKD patients. A recent study reported that higher ACR was associated with LV mass, size, systolic function, and diastolic function in CKD patients [37]. This finding is in agreement with a previous international collaborative study showing a similar pattern for clinical risk of heart failure [38]. Even though the exact mechanisms behind the close link between albuminuria and altered LV structure and function are not clear, this may reflect the property of albuminuria as an indicator of systemic vascular damage, endothelial dysfunction, and microvascular injury in CKD patients [39].

Various abnormalities of mineral-bone metabolism are common in CKD patients, and mineral metabolism disorders such as hypocalcemia, hyperphosphatemia, and vitamin D deficiency have been found to be closely associated with CVD in CKD patients [40]. The mean serum calcium 
and phosphorus levels in the subjects of the present study were within the normal ranges, but differed between the groups with and without LVH. Serum iPTH level was elevated in patients with $\mathrm{LVH}$ and differed from that in the group without LVH. Hypocalcemia was associated with LVH by multivariate logistic regression analysis. Although its mechanism is not completely known, hypocalcemia followed by vitamin D deficiency may be associated with the pathogenesis of LVH. The results of the present study suggested that disorders of mineral metabolism may be involved in the etiology of LVH.

Some limitations have to be acknowledged in this study. First, selection bias cannot be ruled out, because patients were mostly enrolled at large-sized hospitals that can provide nephrology care [41]. Therefore, patients with greater awareness of their treatment probably were selected. Hence, the results from this study might not be applicable to the CKD patients who do not undergo appropriate treatment, are not managed at medical institutions, or do not realize that they have CKD because of the lack of prior medical examination [9]. Second, it is likely that there are possible biases of each study center in the performance of UCG. UCG was performed in most of the patients (80-90\%) of each study center and tended to be underwent for high-risk patients. Thus, there are few selection biases for UCG performance in each study center. Third, because more than $90 \%$ of the patients were treated with ACR inhibitors and/or ARBs, serum potassium levels may be different between patients with and without these medications. Indeed, serum potassium levels $(4.63 \mathrm{mEq} / \mathrm{l})$ in patients with ACE inhibitors and/or ARBs were not significantly different from those $(4.61 \mathrm{mEq} / \mathrm{l})$ in patients with ACE inhibitors and/or ARBs $(P=0.55)$. Fourth, the definition of LVH in Japanese patients may be different from those in the USA. The LVMI value is dependent on body height and body weight. The cutoff values of LVMI were $125 \mathrm{~g} / \mathrm{m}^{2}$ for men and $110 \mathrm{~g} / \mathrm{m}^{2}$ for women in this study. These values were considered as 75 percentile of LVMI in this study. However, it is difficult to determine if the cutoff values of LVMI are suitable for use with the Japanese population. By contrast, the strengths of this study include its multicenter nature, the standard nephrology care, and the evaluation of patients with a wide range of eGFR.

In conclusion, the results of this study showed that the prevalence of LVH was low in stage 3-5 CKD patients treated by nephrologists in Japan. The cross-sectional baseline data from the CKD-JAC study shed light on the association between $\mathrm{LVH}$ and risk factors in patients with decreased renal function. Differences in the presence of previous CVD, blood pressure control, and metabolic state may lead to different outcomes of CVD in a longitudinal study. Future analysis of the CKD-JAC cohort will clarify whether the incidence of LVH varies with the causative disease during further follow-up.

Acknowledgements This study was conducted by the principal investigators at the following medical centers: Yoshio Taguma; Sendai Social Insurance Hospital (Miyagi), Yoshitaka Maeda; JA Toride Medical Center (Ibaragi), Eiji Kusano; Jichi Medical University (Tochigi), Yasuhiro Komatsu; St. Luke's International Hospital (Tokyo), Tadao Akizawa; Showa University Hospital (Tokyo), Eriko Kinugasa; Showa University Yokohama Northern Hospital (Kanagawa), Ashio Yoshimura; Showa University Fujigaoka Hospital (Kanagawa), Hiroshige Ohashi, Hiroshi Oda; Gifu Prefectural General Medical Center (Gifu), Yuzo Watanabe; Kasugai Municipal Hospital (Aichi), Daijo Inaguma, Kei Kurata; Tosei General Hospital (Aichi), Yoshitaka Isaka; Osaka University Hospital (Osaka), Yoshiharu Tsubakihara; Osaka General Medical Center (Osaka), Masahito Imanishi; Osaka City General Hospital (Osaka), Masaki Fukushima; Kurashiki Central Hospital (Okayama), Hideki Hirakata; Fukuoka Red Cross Hospital (Fukuoka), Kazuhito Takeda; Iizuka Hospital (Fukuoka).

\section{Compliance with ethical standards}

Conflict of interest T.A. has consulted for and received lecture fees from Kyowa Hakko Kirin. K.N. has consulted for and received a research support grant from Kyowa Hakko Kirin. S.I., E.I., S.M., H.M., T.W., Y.O. and A.H. have no conflicts of interest to declare.

Ethical approval All procedures performed in studies involving human participants were in accordance with the ethical standards of the institutional and/or national research committee at the institutes where the studies were conducted and with the 1964 Helsinki Declaration and its later amendment or comparable ethical standards. The IRB number was 1106 .

Informed consent Informed consent was obtained from all individual participants included in the study.

Open Access This article is distributed under the terms of the Creative Commons Attribution 4.0 International License (http://creativeco mmons.org/licenses/by/4.0/), which permits unrestricted use, distribution, and reproduction in any medium, provided you give appropriate credit to the original author(s) and the source, provide a link to the Creative Commons license, and indicate if changes were made.

\section{Appendix: Contributors}

1. Steering Committee: Akira Hishida (Yaizu City Hospital), SeiichiMatsuo (Nagoya University), Tsuyoshi Watanabe (Fukushima Rosai Hosapital), Yasuo Ohashi (Chuo University), Hirofumi Makino (Okayama University), Tadao Akizawa (Showa University), Kosaku Nitta (Tokyo Women's Medical University), Enyu Imai (Nakayamadera Imai Clinic)

2. Data Center: Public Health Research Foundation (Tokyo)

3. Independent Cardiac Function Evaluation Committee: Kyoichi Mizuno (Nippon Medical School Hospital), 
Hiroshi Nishimura (The University of Tokyo), Takeo Okada (Osaka Medical Center for Health Science and Promotion), Satoshi Iimuro (The University of Tokyo)

4. Biostatistics Adviser: Yasuo Ohashi (The University of Tokyo)

5. Medical Economics Adviser: Takashi Fukuda (The University of Tokyo)

6. Nutrition Evaluation Adviser: Satoshi Sasaki (The University of Tokyo)

7. International Adviser: Harold I Feldman (University of Pennsylvania)

8. General Adviser: Kiyoshi Kurokawa (National Graduate Institute for Policy Study).

9. Sponsor: Kyowa-Hakko-Kirin Co. Ltd.

\section{References}

1. National Kidney Foundation. K/DOQI clinical practice guidelines for chronic kidney disease: evidence, classification, and stratification. Am J Kidney Dis. 2002;39(suppl 1):1-266.

2. Masakane I, Nakai S, Ogata S, Kimata N, Hanafusa N, Hamano T, et al. Annual Dialysis Data Report 2014, JSDT Renal Data Registry (JRDR). Ren Replace Ther. 2017;3:18.

3. Imai E, Horio M, Watanabe T, Iseki K, Yamagata K, Hara S, et al. Prevalence of jsdt.or.jpchronic kidney disease in the Japanese general population. Clin Exp Nephrol. 2009;13:621-30.

4. Imai E, Horio M, Iseki K, Yamagata K, Watanabe T, Hara S, et al. Prevalence of chronic kidney disease (CKD) in the Japanese general population predicted by the MDRD equation modified by a Japanese coefficient. Clin Exp Nephrol. 2007;11:156-63.

5. Poulikakos D, Ross L, Reico-Mayoral A, Cole D, Andoh J, Chitalia $\mathrm{N}$, et al. Left ventricular hypertrophy and endothelial dysfunction in chronic kidney disease. Eur Heart J Cardiovasc Imaging. 2014;15:56--61.

6. Go AS, Chertow GM, Fan D, McCulloch CE, Hsu CY. Chronic kidney disease and the risks of death, cardiovascular events, and hospitalization. N Engl J Med. 2004;351:1296-305.

7. Ninomiya T, Kiyohara Y, Kubo M, Tanizaki Y, Doi Y, Okubo $\mathrm{K}$, et al. Chronic kidney disease and cardiovascular disease in a general Japanese population: the Hisayama Study. Kidney Int. 2005;68:228-36.

8. Irie F, Iso H, Sairenchi T, Fukasawa N, Yamagishi K, Ikehara S, et al. The relationships of proteinuria, serum creatinine, glomerular filtration rate with cardiovascular disease mortality in Japanese general population. Kidney Int. 2006;69:1264-71.

9. Tanaka K, Watanabe T, Takeuchi A, Ohashi Y, Nitta K, Akizawa $\mathrm{T}$, et al. Cardiovascular events and death in Japanese patients with chronic kidney disease. Kidney Int. 2017;91:227-34.

10. Levin A, Singer J, Thompson CR, Ross H, Lewis M. Prevalent left ventricular hypertrophy in predialysis population: identifying opportunities for intervention. Am J Kidney Dis. 1996;27:347-54.

11. Tucker B, Fabbian F, Giles M, Thuraisingham RC, Raine AE, Baker LR. Left ventricular hypertrophy and ambulatory blood pressure monitoring in chronic renal failure. Nephrol Dial Transplant. 1997;12:724-8.

12. McMahon LP, Roger SD, Slimheart Investigators Group. Development, prevention, and potential reversal of left ventricular hyperterophy in chronic kidney disease. J Am Soc Nephrol. 2004:15:1640-7.

13. Paoletti E, Bellino D, Cassottana P, Rolla D, Cannella G. Left ventricular hypertrophy in nondiabetic predialysis patients. Am J Kidney Dis. 2005;46:320-7.

14. Imai E, Matsuo S, Makino H, Watanabe T, Akizawa T, Nitta K, et al. Chronic Kidney Disease Japan Cohort study: baseline characteristics and factors associated with causative diseases and renal function. Clin Exp Nephrol. 2010;14:558-70.

15. Imai E, Matsuo $S$, Makino $H$, Watanabe $T$, Akizawa $T$, Nitta $K$, et al. Chronic Kidney Disease Japan Cohort (CKD-JAC) study: design and methods. Hypertens Res. 2008;3:1101-7.

16. Matsuo S, Imai E, Horio M, Yasuda Y, Tomita K, Nitta K, et al. Revised equations for estimated GFR from serum creatinine in Japan. Am J Kidney Dis. 2009;53:982-92.

17. Reichek N, Devereux RB. Left ventricular hypertrophy: relationship of anatomic, echocardiographic and electrocardiographic findings. Circulation. 1981;63:1391-8.

18. Devereux RB, Alonso DR, Lutas EM, Gottlieb GJ, Campo E, Sachs I, Reichek N. Echocardiographic assessment of left ventricular hypertrophy: comparison to necropsy findings. Am J Cardiol. 1986;57:450-8.

19. Park M, Hsu CY, Li Y, Mishra RK, Keane M, Rosas SE, et al. Associations between kidney function and subclinical cardiac abnormalities in CKD. J Am Soc Nephrol. 2012;23:1725-34.

20. Yoshitomi R, Fukui A, Nakayama M, Ura Y, Ikeda H, Onuki H, et al. Sex differences in the association between serum uric acid levels and cardiac hypertrophy in patients with chronic kidney disease. Hypertens Res. 2014;37:246-52.

21. Asp AM, Wallquist C, Rickenlund A, Hylander B, Jacobson SH, Caidahl K, et al. Cardiac remodeling and functional alterations in mild-to-moderate renal dysfunction: comparison with healthy subjects. Clin Physiol Funct Imaging. 2015;35:223-30.

22. Miura K, Nakagawa H, Ohashi Y, Harada A, Taguri M, Kushiro $\mathrm{T}$, et al. Four blood pressure indexes and the risk of stroke and myocardial infarction in Japanese men and women: a metaanalysis of 16 cohort studies. Circulation. 2009;119:1892-8.

23. Paoletti E, De Nicola L, Gabbai FB, Chiodini P, Ravera M, Pieracci L, et al. Associations of left ventricular hypertrophy and geometry with adverse outcomes in patients with CKD and hypertension. Clin J Am Soc Nephrol. 2016;11:271-9.

24. Ahmad FS, Cai X, Kunkel K, Ricardo AC, Lash JP, Raj DS, et al. Racial/ethnic differences in left ventricular structure and function in chronic kidney disease: the chronic renal insufficiency cohort. Am J Hypertens. 2017;30:822-9.

25. Levin A, Thrompson CR, Ethier J, Carisie EJ, Tobe S, Mendelssohn D, et al. Left ventricular mass index increase in early renal disease: impact of decline in hemoglobin. Am J Kidney Dis. 1999;34:125-34.

26. Nardi E, Palermo A, Mule G, Cusimano P, Cotton S, Cerasola G. Left ventricular hypertrophy and geometry in hypertensive patients with chronic kidney disease. J Hypertens. 2009;27:633-41.

27. Locatelli F, Bommer J, London GM, Martin-Malo A, Wanner C, Yaqoob M, et al. Cardiovascular disease determinants in chronic renal failure: clinical approach and treatment. Nephrol Dial Transplant. 2001;16:459-68.

28. London G. Pathophysiology of cardiovascular damage in the early renal population. Nephrol Dial Transplant. 2001;16(Suppl 2):3-6.

29. Nitta K. Pathogenesis and therapeutic implications of cardiorenal syndrome. Clin Exp Nephrol. 2011;15:187-94.

30. Alpert MA. Obesity cardiomyopathy: pathophysiology and evolution of the clinical syndrome. Am J Med Sci. 2001;321:225-36.

31. Kotsis V, Stabouli S, Toumanidis S, Tsivqoulis G, Rizos Z, Trakateli $\mathrm{C}$, et al. Obesity and daytime pulse pressure are 
predictors of left ventricular hypertrophy in true normotensive individuals. J Hypertens. 2010;28:1065-73.

32. Guerra F, Mancinelli L, Angelini L, Fortunati M, Rappelli A, Dessi-Fulgheri $\mathrm{P}$, et al. The association of left ventricular hypertrophy with metabolic syndrome is dependent on body mass index in hypertensive overweight or obese patients. PLoS One. 2011;6:e16630.

33. Verhave JC, Hillege HL, Burgerhof JG, Navis G, de Zeeuw D, de Jong PE, et al. Cardiovascular risk factors are differently associated with urinary albumin excretion in men and women. J Am Soc Nephrol. 2003;14:1330-5.

34. Meisinger C, Doring A, KORA Study Group. Chronic kidney disease and risk of incident myocardial infarction and all-cause and cardiovascular disease mortality in middle-aged men and women from the general population. Eur Heart J. 2006;27:1245-50.

35. Kurth T, de Jong PE, Cook NR, Buring JE, Ridker PM. Kidney function and risk of cardiovascular disease and mortality in women: a prospective cohort study. BMJ. 2009;338:b2392.

36. Muiesan ML, Ambrosioni E, Costa FV, Leonetti G, Pessina AC, Salvetti M, et al. Sex differences in hypertension-related renal and cardiovascular disease in Italy: the I-DEMAND study. J Hypertens. 2012;30:2378-86.
37. Matsushita K, Kwak L, Sang Y, Ballew SH, Skali H, Shah AM, et al. Kidney disease measures and left ventricular structure and function: The Atherosclerosis Risk in Community Study. J Am Heart Assoc. 2017;6:e006259.

38. Matsushita K, Coresh J, Sang Y, Chalmers J, Fox C, Guallar E, et al. Estimated glomerular filtration rate and albuminuria for prediction of cardiovascular outcomes: a collaborative meta-analysis of individual participant data. Lancet Diabetes Endocrinol. 2015;3:514-25.

39. El Nahas M. Cardio-Kidney-Damage: a unifying concept. Kidney Int. 2010;78:14-8.

40. Covic A, Kothawala P, Nernal M, Robbins S, Chalian A, Goldsmith D. Systematic review of the evidence underlying the association between mineral metabolism disturbances and risk of all-cause mortality, cardiovascular mortality and cardiovascular events in chronic kidney disease. Nephrol Dial Transplant. 2009;24:1506-23.

41. Inaguma $D$, Imai $E$, Takeuchi $A$, Ohashi $Y$, Watanabe $T$, Nitta $K$, et al. Risk factors for CKD progression in Japanese patients: findings from the Chronic Kidney Disease Japan Cohort (CKD-JAC) study. Clin Exp Nephrol. 2017;21:446-56. 